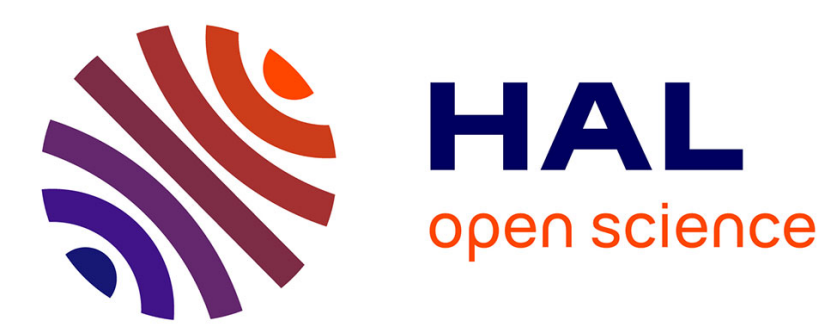

\title{
First-principles calculations of threading screw dislocations in AIN and InN
}

Laurent Pizzagalli, I. Belabbas, J. Kioseoglou, Jun Chen

\section{To cite this version:}

Laurent Pizzagalli, I. Belabbas, J. Kioseoglou, Jun Chen. First-principles calculations of threading screw dislocations in AlN and InN. Physical Review Materials, 2018, 2 (6), 10.1103/PhysRevMaterials.2.064607 . hal-02114776

\section{HAL Id: hal-02114776 \\ https://hal.science/hal-02114776}

Submitted on 29 Apr 2019

HAL is a multi-disciplinary open access archive for the deposit and dissemination of scientific research documents, whether they are published or not. The documents may come from teaching and research institutions in France or abroad, or from public or private research centers.
L'archive ouverte pluridisciplinaire HAL, est destinée au dépôt et à la diffusion de documents scientifiques de niveau recherche, publiés ou non, émanant des établissements d'enseignement et de recherche français ou étrangers, des laboratoires publics ou privés. 


\title{
First-principles calculations of threading screw dislocations in AIN and InN
}

\author{
L. Pizzagalli, ${ }^{1, *}$ I. Belabbas, ${ }^{2, \dagger}$ J. Kioseoglou, ${ }^{3}$ and J. Chen ${ }^{4}$ \\ ${ }^{1}$ Department of Physics and Mechanics of Materials, Institut P', CNRS-Université de Poitiers UPR 3346, SP2MI, BP 30179, \\ F-86962 Futuroscope Chasseneuil Cedex, France \\ ${ }^{2}$ Equipe de Cristallographie et de Simulation des Matériaux, Laboratoire de Physico-chimie des Matériaux et Catalyse, \\ Faculté des Sciences Exactes, Université de Bejaia, 06000 Bejaia, Algeria \\ ${ }^{3}$ Department of Physics, Aristotle University of Thessaloniki, GR-54124 Thessaloniki, Greece \\ ${ }^{4}$ CIMAP-Alençon, UMR6252, CNRS-CEA-ENSICAEN, Université de Caen Normandie, F-14032, France
}

(Received 24 May 2018; published 29 June 2018)

\begin{abstract}
The threading screw dislocation in AlN and InN, two technologically important nitride-based materials, has been studied using first-principles calculations. By performing a comprehensive search for the most stable core configuration, several original core structures have been obtained depending on experimental conditions. In Al-rich and In-rich conditions, screw dislocation cores exhibit a metalliclike character, predicted to provide pathways to leakage currents in III-N based electronic devices. Specifical to AlN, most stable core geometries in N-rich conditions are shown to contain nitrogen atoms organized like nitrogen molecules. Finally, our investigations revealed that in InN dislocation cores are associated to partial or complete closure of the gap and to the injection of electrons into the bulk conduction band.
\end{abstract}

DOI: 10.1103/PhysRevMaterials.2.064607

\section{INTRODUCTION}

III-nitride binary compounds GaN, AlN, and InN, and their alloys, form an important class of materials, at the core of numerous optoelectronic applications. The critical property is their direct band gap with energies allowing for light-emitting devices (LED) and detectors in a spectral range from near infrared to deep ultraviolet (for a recent review, see [1]). GaN blue-light LEDs probably represent the most famous successful example of applications, but III-nitrides are also prospects for technological developments in numerous other domains such as biochemistry, electronics, medicine, solar energy conversion, etc. The efficiency of III-nitrides based devices usually strongly depends on the crystalline quality of the materials, and there is an intense research activity devoted to reducing the presence of structural defects and impurities through diverse strategies. Active layers in devices are typically obtained by heteroepitaxial growth on substrates like sapphire or silicon. Due to lattice parameters and thermal expansion coefficients mismatches, threading dislocations can hardly be avoided. These defects can act as nonradiative recombination centers, thus decreasing the light emitting efficiency. They can also induce segregation and diffusion of impurities and dopants. Furthermore, it has also been shown that dislocations could exhibit a metallic character along the dislocation line, thus acting as a pathway for leakage current [2-4].

The detrimental influence of threading dislocations motivated numerous investigations, both experimentally and theoretically, mainly on gallium nitride. In particular, edge dislocations have been extensively studied, because their influence

\footnotetext{
*laurent.pizzagalli@univ-poitiers.fr

†imad.belabbas@univ-bejaia.dz
}

on electronic properties, and by extension on the performance of light-emitting devices, triggered a long time debate. Three possible structures have been identified for the edge dislocation $[5,6]$, and it is commonly agreed that all three could exist in $\mathrm{GaN}$, depending on experimental conditions and the presence of dopants [7-13]. For edge dislocations in other nitride materials, the literature is rather limited, although there have been investigations on AIN [14], InN [15,16], and ternary nitride alloys [17].

The prismatic screw dislocation received less attention in comparison, and its influence on electronic properties is not fully understood [18]. In a pioneering study, Elsner and co-workers reported an open-core configuration, centered on one hexagon, as the most stable state $[5,19]$. Next, this result was confirmed by Northrup, although he also proposed an alternative $\mathrm{Ga}$-filled core, lower in energy in Ga-rich conditions [20,21]. A few years later, atomistic calculations suggested that a new dislocation core structure, centered on a $\mathrm{Ga}-\mathrm{N}$ bond, could be more stable [10,22]. At last, more recent investigations by Matsubara and co-workers revealed that, in N-rich conditions, the lowest energy structure would be obtained by removing atoms from this bond-centered core $[23,24]$.

This is a good illustration of the main difficulty with nitride materials, that is the variety of possible dislocation core geometries, in particular when considering that dislocation cores could be nonstoichiometric. Yet it is a prerequisite to determine the structure of these dislocation cores, before trying to understand how dislocations could influence electronic properties, or trap defects or dopants. Unfortunately, for important materials such as AlN and InN, our knowledge remains scarce by lack of dedicated studies. As far as we know, Kioseoglou et al. investigated two possible stoichiometric screw configurations in AlN [25]. More recently, a single configuration was tested 
in AlGaN and InGaN [17]. Not all dislocation geometries revealed by works on GaN have been studied in AlN and InN. It is also not possible to exclude that new stable configurations could be found for these two materials.

To address this issue, we have performed a comprehensive study of prismatic screw dislocations in AlN and InN, using state of the art first-principles calculations, including the use of a hybrid exchange correlation functional. In particular, we have investigated a large number of possible configurations for dislocation cores, taking into account nonstoichiometry. These calculations revealed that, in AlN ( $\mathrm{InN})$, the lowest energy configuration for Al-rich (In-rich) conditions is an Al-filled (In-filled) hexagon-centered structure, respectively. For AlN and intermediate growth conditions, an open bond-centered geometry is found to be the most stable, like for GaN. Two original configurations, with $\mathrm{N}$-filled cores, are discovered in this work in N-rich conditions and for AlN. A different picture is obtained for $\mathrm{InN}$, since two bond-centered geometries, with either a N-filled or a full stoichiometric core, are found to be of low energy for intermediate and N-rich conditions, respectively. Both atomic and electronic structures of all these most stable dislocation cores are described.

In the remainder of this paper, the first-principles calculations are first detailed, followed by a description of the model and how dislocation core initial geometries are generated. The energetics of all calculated geometries are then reported in a third section. The next two sections deal with a description of the atomic and electronic structures of the most stable configurations. Our main findings are then discussed, followed by a concluding section.

\section{METHODOLOGY}

Our calculations have been carried out in the framework of density functional theory with wave functions developed on a plane-waves basis set. In the case of aluminum nitride, the Quantum Espresso [32] package has been used for performing calculations. Exchange and correlation contributions were computed within the generalized gradient approximation while using the Perdew-Burke-Ernzerhof (GGA-PBE) functional [33], and the electron-ion interactions were described by employing the full-potential projector augmented wave (PAW) method [34]. A plane-waves cutoff of $544.2 \mathrm{eV}$ was found to be sufficient for an accurate determination of the properties of bulk AlN in the wurtzite structure (Table I). The small

TABLE I. Lattice constants ( $a_{0}$ and $c_{0}$, in $\AA$ ), enthalpy of formation $\left(\Delta H_{f}^{0}\right.$, in eV), and electronic band gap $\left(E_{g}\right.$, in eV), for bulk $\mathrm{AlN}$ and InN, compared to reference values. AlN values have been calculated using GGA-PBE except for the band gap with is determined using the HSE06 functional. InN values have been calculated using LDA.

\begin{tabular}{lllll}
\hline \hline & \multicolumn{1}{c}{$a_{0}(\AA)$} & \multicolumn{1}{c}{$c_{0}(\AA)$} & \multicolumn{1}{c}{$\Delta H_{f}^{0}(\mathrm{eV})$} & \multicolumn{1}{c}{$E_{g}(\mathrm{eV})$} \\
\hline AlN & 3.127 & 5.013 & -2.80 & 5.28 \\
Ref. & $3.11[26]$ & $4.98[26]$ & $-(3.23-3.32)[27,28]$ & $6.03[29]$ \\
InN & 3.5542 & 5.7205 & -0.778 & 0.85 \\
Ref. & $3.537[27]$ & $5.704[27]$ & $-(0.18-1.52)[27,30]$ & $0.7[31]$ \\
\hline \hline
\end{tabular}

overestimation of lattice parameters compared to experiments is usual for GGA-PBE calculations and well documented in the literature [35]. We also determined the heat of formation of AlN, by comparing the total energies of bulk AlN and $\mathrm{Al}$, and of a $\mathrm{N}_{2}$ molecule. The calculated value is $12 \%-15 \%$ lower than in experiments, which is also a known feature of semilocal exchange correlation functionals [36]. Note also that thermal and zero-point contributions are lacking in our calculations, whereas experimental values are obtained at room temperature.

The inability of DFT-GGA to reproduce electronic band gaps is well known, and one possible way to circumvent this issue is to use hybrid functionals. In this work, we determine the electronic structure of the dislocation cores by performing subsequent calculations with the Heyd-Scuseria-Ernzerhof (HSE06) hybrid functional [37,38]. Since the computational cost of the latter is much higher than standard GGA-PBE calculations, only the electronic structure is computed, with no additional ionic relaxations. One of us has previously shown that this approach leads to negligible errors in the case of dislocations in GaN [23]. The wurtzite bulk AlN band gap computed with HSE06, reported in Table I, is about $12 \%$ lower than the experimental value. This is to be contrasted with standard GGA-PBE calculations which underestimate the experimental value by $33 \%$.

Indium nitride calculations have been carried out using the ABINIT code [39]. In this particular case, we have taken advantage of the existence of specifically designed local density approximation (LDA) pseudopotentials [40], which can accurately reproduce the electronic band gap of indium nitride. For that purpose norm-conserving Trouiller-Martins pseudopotentials have been utilized [41]. The PW92 exchange and correlations functional was adopted [42]. We have included In-4 $d$ electrons in the valence and we found that a planewaves energy cutoff of $952.4 \mathrm{eV}$ was a good compromise between accuracy and computational cost. Table I reports the calculations results for InN of the same quantities as for AIN. Obviously, both lattice constants and electronic band gap are in excellent agreement with reference data. For the formation enthalpy, although an experimental consensus is lacking, our calculated value lies in the middle of the measurements range [27,30].

Since we aim at investigating dislocations, it is equally important to assess the reliability of our computational setup for mechanical properties. In Table II, we compare the calculated elastic constants with data available in the literature. For AlN, we obtained values slightly lower than experiments, but overall in good agreement with experiments. In the case of InN, there is an important dispersion among published data, because it is difficult to grow this material without defects and with a

TABLE II. Calculated elastic constants (in GPa) for bulk AlN and InN, compared to the literature.

\begin{tabular}{lccccc}
\hline \hline & $\mathrm{C}_{11}$ & $\mathrm{C}_{12}$ & $\mathrm{C}_{13}$ & $\mathrm{C}_{33}$ & $\mathrm{C}_{44}$ \\
\hline AlN & 378 & 126 & 98 & 359 & 113 \\
Ref. [43] & $345-410$ & $125-148$ & $99-120$ & $388-395$ & $118-125$ \\
InN & 232 & 124 & 106 & 232 & 49 \\
Refs. [44,45] & $190-298$ & $85-120$ & $70-121$ & $182-278$ & $10-89$ \\
\hline \hline
\end{tabular}


width sufficient enough to carry out dedicated experiments. Nonetheless, our calculated values are in the range of reported data. The information gathered in Tables I and II indicates that our computational setup is appropriate to compute dislocation core properties in AlN and InN.

Due to the computational cost of first-principles calculations, especially when using hybrid functionals, those are necessarily restricted to small system sizes. It is a critical point in the case of the present calculations, since a dislocation is a topological defect that induces a long range deformation field. Several techniques have been proposed to deal with this issue [46], each one having advantages and drawbacks. Here we have chosen full periodic boundary conditions, for which the dislocation displacement field is perfectly defined at the boundaries. However, this setup implies an elastic interaction between dislocations.

Our supercells are defined according to the following $\mathbf{c}_{\mathbf{i}}$ vectors: $\mathbf{c}_{\mathbf{1}}=n_{1} a_{0} \hat{\mathbf{x}}, \mathbf{c}_{\mathbf{2}}=n_{1} a_{0} / 2 \hat{\mathbf{x}}+n_{2} \sqrt{3} a_{0} \hat{\mathbf{y}}+c_{0} / 2 \hat{\mathbf{z}}$, and $\mathbf{c}_{3}=c_{0} \hat{\mathbf{z}}$, with $\hat{\mathbf{x}}=1 / 3[\overline{1} 2 \overline{1} 0], \hat{\mathbf{y}}=1 / \sqrt{3}[\overline{1} 010]$, and $\hat{\mathbf{z}}=$ [0001]. This specific geometry has been demonstrated to be optimal to model screw dislocations, with minimal elastic interaction $[47,48]$. Two screw dislocations of opposite Burgers vectors $\left(\mathbf{b}= \pm c_{0} \hat{\mathbf{z}}\right)$ are inserted in this supercell with a separation $n_{1} a_{0} / 2$ along $\hat{\mathbf{x}}$, by using the atomic displacements as given by elasticity theory [49]. Applying periodic boundary conditions results in an infinite quadrupolar array of dislocations. The supercell shape and dimensions remain fixed during relaxation. To ensure this has a negligible effect on dislocation core properties, two different supercell sizes have been used in this work. The first one is obtained with $\left(n_{1}=8, n_{2}=2\right)$, and the second one with $\left(n_{1}=12, n_{2}=3\right)$, encompassing 128 and 288 atoms, respectively. For both systems, the Brillouin zone is sampled using a $1 \times 1 \times 4 k$-points grid [50].

In our calculations, ions were relaxed by using the BroydenFletcher-Goldfarb-Shanno algorithm, with a maximum force criterion of $2.6 \times 10^{-2} \mathrm{eV} / \AA$. About half of the calculations have been performed with a stricter criterion of $2.1 \times$ $10^{-3} \mathrm{eV} / \AA$. The two levels of convergence were compared for selected configurations by computing the root mean square deviation over all atomic coordinates. We found that this quantity is lower than $0.002 \AA$, indicating that well relaxed structures are already obtained using the loose force criterion.

After relaxation, we determine the stability of the different dislocation core configurations by comparing their formation energies $E_{f}$. Taking AlN as an example, $E_{f}$ is defined as

$$
\begin{aligned}
E_{f} & =E-n_{\mathrm{N}} \mu_{\mathrm{N}}-n_{\mathrm{Al}} \mu_{\mathrm{Al}} \\
& =E-E^{\circ}+\left(n_{\mathrm{N}}^{\circ}-n_{\mathrm{N}}\right) \mu_{\mathrm{N}}+\left(n_{\mathrm{Al}}^{\circ}-n_{\mathrm{Al}}\right) \mu_{\mathrm{Al}} \\
& =E-E^{\circ}+\Delta n_{\mathrm{N}} \mu_{\mathrm{N}}+\Delta n_{\mathrm{Al}} \mu_{\mathrm{Al}},
\end{aligned}
$$

where $E$ is the total energy of the supercell with dislocations, $E^{\circ}$ is the bulk total energy using the same supercell, $\mu_{\mathrm{Al}}$ and $\mu_{\mathrm{N}}$ are the chemical potentials of $\mathrm{Al}$ and $\mathrm{N}$ in $\mathrm{AlN}$, and $n_{\mathrm{Al}}\left(n_{\mathrm{N}}\right)$ and $n_{\mathrm{Al}}^{\circ}\left(n_{\mathrm{N}}^{\circ}\right)$ are the numbers of $\mathrm{Al}(\mathrm{N})$ atoms in the systems with and without the dislocations, respectively. The chemical potentials obey the following equations:

$$
\begin{aligned}
\mu_{\mathrm{AlN}} & =\mu_{\mathrm{Al}}+\mu_{\mathrm{N}} \\
& =\mu_{\mathrm{Al}}^{\circ}+\mu_{\mathrm{N}}^{\circ}+\Delta H_{f}^{0},
\end{aligned}
$$

with $\Delta H_{f}^{0}$ the formation enthalpy of $\mathrm{AlN}, \mu_{\mathrm{Al}}^{\circ}$ the chemical potential of bulk aluminum, and $\mu_{\mathrm{N}}^{\circ}$ the chemical potential of $\mathrm{N}$ in the $\mathrm{N}_{2}$ molecule (calculated at $0 \mathrm{~K}$ ). The expression (5) defines the range of thermodynamically allowed values for the chemical potentials. For instance, $\mu_{\mathrm{Al}}$ ranges from $\mu_{\mathrm{Al}}^{\circ}+$ $\Delta H_{f}^{\circ}$ to $\mu_{\mathrm{Al}}^{\circ}$. Note that, experimentally, the chemical potential values are set by the growth conditions of the material. For AlN, we used the experimental $\Delta H_{f}^{\circ}$ value since accurate data are available (Table I). Conversely, the calculated value is used for InN because of the lack of a well established experimental reference.

Since $\mu_{\mathrm{N}}$ can be expressed as a function of $\mu_{\mathrm{Al}}, E_{f}$ is here computed as a function of $\mu_{\mathrm{Al}}$ according to the equation

$$
E_{f}=E-E^{\circ}+\Delta n_{\mathrm{N}}\left(\mu_{\mathrm{AlN}}-\mu_{\mathrm{Al}}\right)+\Delta n_{\mathrm{Al}} \mu_{\mathrm{Al}} .
$$

The same formalism is used to compute the dislocation core formation energies in the case of $\mathrm{InN}$, with $E_{f}$ computed as a function of $\mu_{\mathrm{In}}$. Note that due to the small sizes of the supercell used in our first-principles calculations, computed formation energies include a non-negligible contribution due to the interaction between all dislocations in the quadrupolar array. However, this interaction is mostly elastic and is assumed to remain the same for all configurations. It is then possible to determine the stability by comparing the formation energies. We tested the validity of this approach by using two different supercell sizes. Finally, additional tests on few AlN dislocation core configurations were also performed to estimate the accuracy of electronic structure calculations. Considering either a larger plane-waves cutoff equal to $816.34 \mathrm{eV}$ or a finer $1 \times 1 \times 8 k$-point sampling, formation energy differences of $30 \mathrm{meV}$ and $3 \mathrm{meV}$ were obtained, respectively.

\section{MODELS}

Defects in covalently bonded materials are generally characterized by an abundance of possible atomic structures, because bonds in the defect core can reconstruct in multiple ways. This point makes more difficult the search for the most stable configuration. Chances of success clearly increase by considering as many as possible candidates though. In this work, we consider 14 different initial configurations, for both AlN and InN.

These initial structures are generated according to the following procedure. First, different core geometries can be obtained depending on how the elastic displacement field is positioned relative to the wurtzite lattice. It is common to center the field on specific positions, usually chosen as far as possible from lattice atoms in order to minimize the strain in the core. For instance, the center of a hexagon has been considered in the earlier studies of dislocations in wurtzite $\mathrm{GaN}$ [21]. Later, also for $\mathrm{GaN}$, it has been proposed to center the core on one covalent bond [10,22]. Both cases have been studied in this work, the former being named A and the latter B (Fig. 1). Different groups have attempted to perform a systematic search for new prismatic screw core configurations in $\mathrm{GaN}$ by varying the origin of the displacement field [51,52]. These studies did not reveal new core structures with a drastically different geometry compared to previously reported configurations. It is also noteworthy that simply relaxing the ionic positions given by the elastic displacement field could not always reveal the 


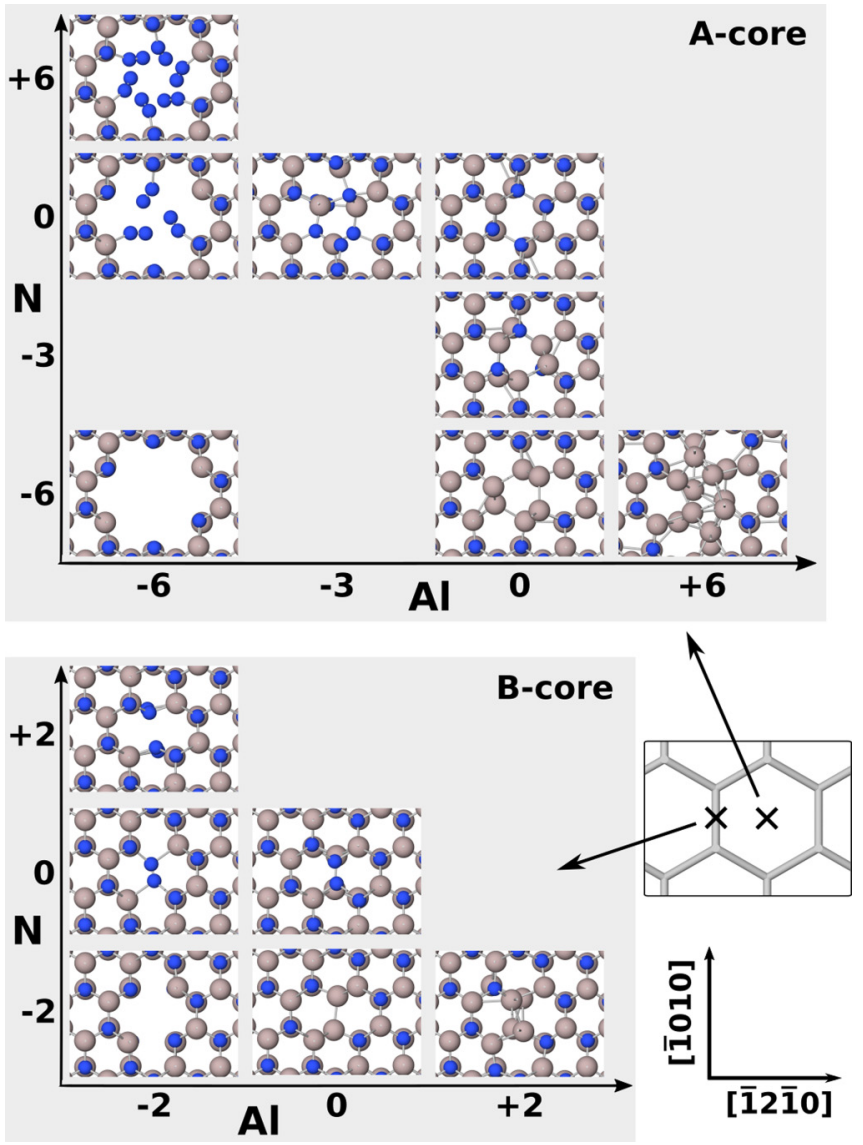

FIG. 1. Various relaxed AIN dislocation core configurations investigated in this work. Top panel: configurations corresponding to dislocation center in A position, in the center of one hexagon (basal plane projection). Bottom: configurations in B position, centered on one bond. In both cases, different core stoichiometries have been considered (indicated along "Al" and "N" axis), (0|0) corresponding to the stoichiometric full core (no $\mathrm{Al} / \mathrm{N}$ atoms added or removed).

energetically most stable structure. For instance, it is known that the nondissociated $60^{\circ}$ shuffle dislocation core in silicon cannot be obtained directly by this approach [53].

Next, starting from the structures directly issued from the elastic displacement field, the local composition of the core is modified to generate new configurations. In fact a substantial energy gain could be obtained if high energy core atoms, involved in strongly distorted covalent bonds, are removed $[21,23,24]$. Also, it could be energetically favored to alter the stoichiometry of the core, by removing (or adding) only atoms from one species. In that case, the stability of the configurations will depend on the chemical potential values.

In this work, we consider as many as 14 different screw dislocation cores for AlN and $\mathrm{InN}$, depending on the structure and composition (Fig. 1). They are named A or B (dislocation core center), followed by the number of $\mathrm{Al}$ (In) and $\mathrm{N}$ atoms added or removed. For instance, the configuration $\mathrm{B}(-2 \mid+2)$ corresponds to a bond-centered core, with two removed $\mathrm{Al}$ (In) atoms and two added $\mathrm{N}$ atoms.

Finally, we did not consider the influence of charge on the properties of the screw dislocation, for two reasons. The first is that we primarily focus on the core composition, with

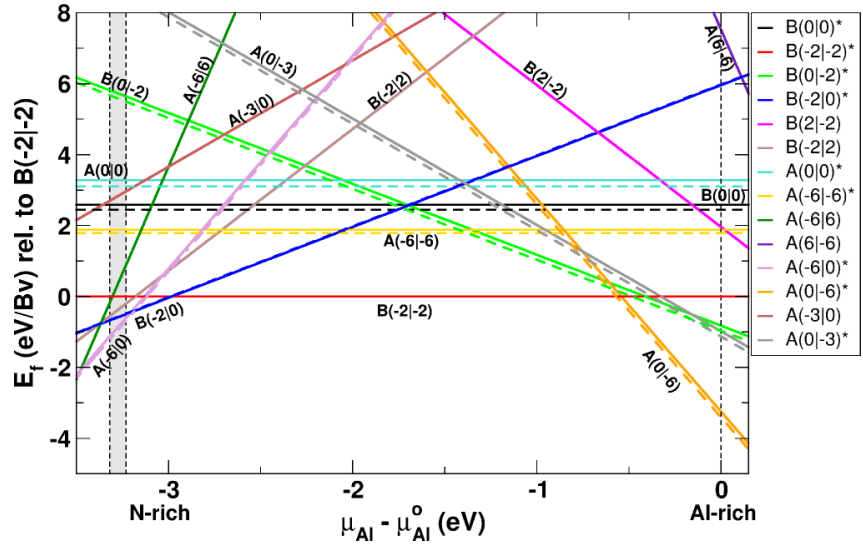

FIG. 2. Formation energy differences between various screw dislocation core structures in AlN, with configuration $\mathrm{B}(-2 \mid-2)$ chosen as the reference, as a function of the $\mathrm{Al}$ chemical potential. Full (dashed) lines correspond to calculations performed in small (large) supercells. Configurations marked by an asterisk have been relaxed in both the 128- and 288-atoms supercells. The gray area shows the experimental range of possible lowest chemical potential values, from $\mu_{\mathrm{Al}}-\mu_{\mathrm{Al}}^{\circ}=-3.32 \mathrm{eV}$ to $-3.23 \mathrm{eV}$ (Table I).

an already large number of candidate configurations. The second is related to the fact that prismatic screw dislocations form during material growth, then before a potential doping. Although the latter could modify the structure and stability of the screw dislocation core, it is also probable that the configuration formed during growth would be quenched. Nonetheless, determining the influence of charge should motivate future investigations.

\section{ENERGETICS AND STABILITY}

We start with the description of the stability of the various calculated core structures in AlN, as a function of the Al chemical potential. Figure 2 shows the formation energy differences between all those configurations. For the highest $\mu_{\mathrm{Al}}$ value, i.e., in Al-rich conditions, the lowest energy configuration is $A(0 \mid-6)$, represented in Figs. 1 and 4. It is obtained by removing the nitrogen atoms in the core. This configuration is equivalent to the Ga-filled configuration reported as the most stable core geometry in GaN [20,21], for Ga-rich conditions.

Decreasing $\mu_{\mathrm{Al}}$ below $\mu_{\mathrm{Al}}^{\circ}-0.55 \mathrm{eV}$, the next most stable configuration becomes $\mathrm{B}(-2 \mid-2)$ (Fig. 1). Here the bondcentered dislocation core is open, but with a smaller empty volume than in the case of the well known hexagon-centered configuration [5]. This core has also been reported as the most stable one in $\mathrm{GaN}$, but for N-rich conditions [23,24]. This core geometry has the lowest energy for a large range of $\mu_{\mathrm{Al}}$. Only when $\mu_{\mathrm{Al}}<\mu_{\mathrm{Al}}^{\circ}-2.96 \mathrm{eV}$, a new core geometry, $\mathrm{B}(-2 \mid 0)$, is obtained. This configuration, seemingly never reported before, is characterized by a bond-centered N-filled core. Finally, for $\mu_{\mathrm{Al}}<\mu_{\mathrm{Al}}^{\circ}-3.19 \mathrm{eV}$, we found another original structure, $\mathrm{A}(-6 \mid 0)$, as the most stable state. In the latter, the hexagoncentered core contains only nitrogen atoms (Figs. 1 and 4).

Figure 2 also reveals the influence of supercell size relative to the formation energy differences. In fact, full (dashed) lines correspond to 128 -atoms (288-atoms) supercell calculations. 


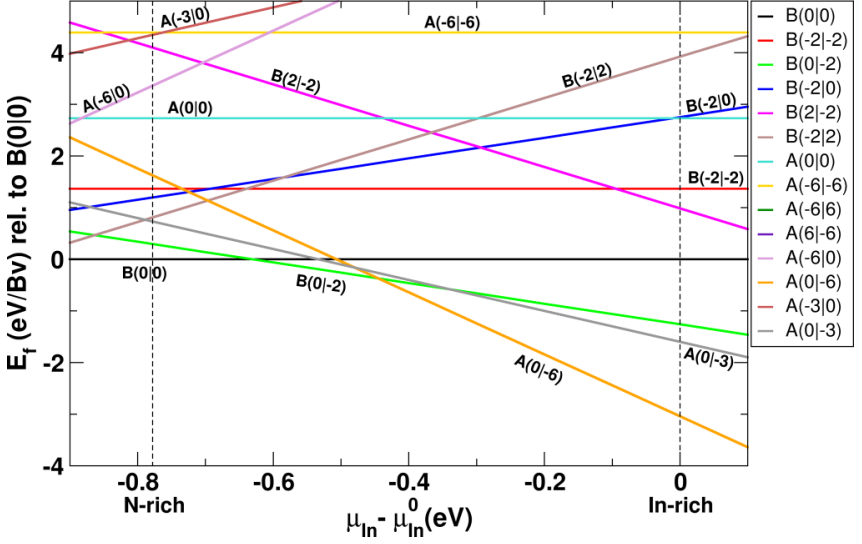

FIG. 3. Formation energy differences between various screw dislocation core structures in $\mathrm{InN}$, with configuration $\mathrm{B}(0 \mid 0)$ chosen as the reference, as a function of the In chemical potential.

Although the size effect depends on the relaxed core geometry, it is always small compared to formation energy differences. This suggests that a 128 -atoms supercell is large enough to determine the properties of the prismatic screw dislocation.

From this conclusion, only 128-atoms supercell calculations have been carried out for InN. Figure 3 shows the formation energy differences as a function of the In chemical potential. In In-rich conditions, the lowest energy configuration is the same as for $\mathrm{AlN}$ and $\mathrm{GaN}$, i.e., the hexagon-centered open core filled with In atoms (or, equivalently, a full core where only $\mathrm{N}$ atoms have been removed). This core is the most stable one until $\mu_{\text {In }}<\mu_{\text {In }}^{\circ}-0.44 \mathrm{eV}$, below which $\mathrm{B}(0 \mid-2)$ becomes energetically favored. In this geometry, the dislocation core is centered on one bond, and is characterized by a central In-In dimer. To our knowledge, this specific configuration has not been reported in the literature. Finally, when $\mu_{\mathrm{In}}<\mu_{\mathrm{In}}^{\circ}-0.64 \mathrm{eV}$, $\mathrm{B}(0 \mid 0)$, i.e., a full bond-centered core, is found to have the lowest energy. This is in stark contrast to $\mathrm{AlN}$ and $\mathrm{GaN}$, for which a bond-centered open core is predicted.

\section{DISLOCATION CORE STRUCTURE}

In this section, we describe the structural features of the most stable dislocation core configurations for $\mathrm{AlN}$ and $\mathrm{InN}$, whose relaxed structures are shown in Fig. 4. For now, atoms are assumed to be bonded according to distance criteria. Starting with AlN and A $(0 \mid-6)$, the analysis of the relaxed configuration reveals a significant reorganization of the aluminum atoms in the dislocation core. Those are connected together according to two types of bonds: one with a length of $2.53 \AA$ and a $\Delta \hat{\mathbf{z}}$ tilt along [0001] of $0.88 \AA$, and the second with a length of $2.73 \AA$ and $\Delta \hat{\mathbf{z}}$ equal to $2.45 \AA$. Each of these $\mathrm{Al}$ core atoms makes a bond with a single $\mathrm{N}$ atom, with bond lengths ranging from 1.91 to $1.96 \AA$, close to the $\mathrm{Al}-\mathrm{N}$ bond distance in bulk AlN $(1.90 \AA)$. The $\mathrm{A}(0 \mid-6)$ core configuration can be viewed as composed of two interwoven helical chains of $\mathrm{Al}$ atoms along the $\hat{\mathbf{z}}$ axis, each one having a period of $2 c_{0}$. Alternatively, one could consider that the $\mathrm{Al}$ atoms are forming three zigzag dimer chains along $\hat{\mathbf{z}}$ on the inner prismatic surfaces of the dislocation core. These dimers are characterized by lengths of
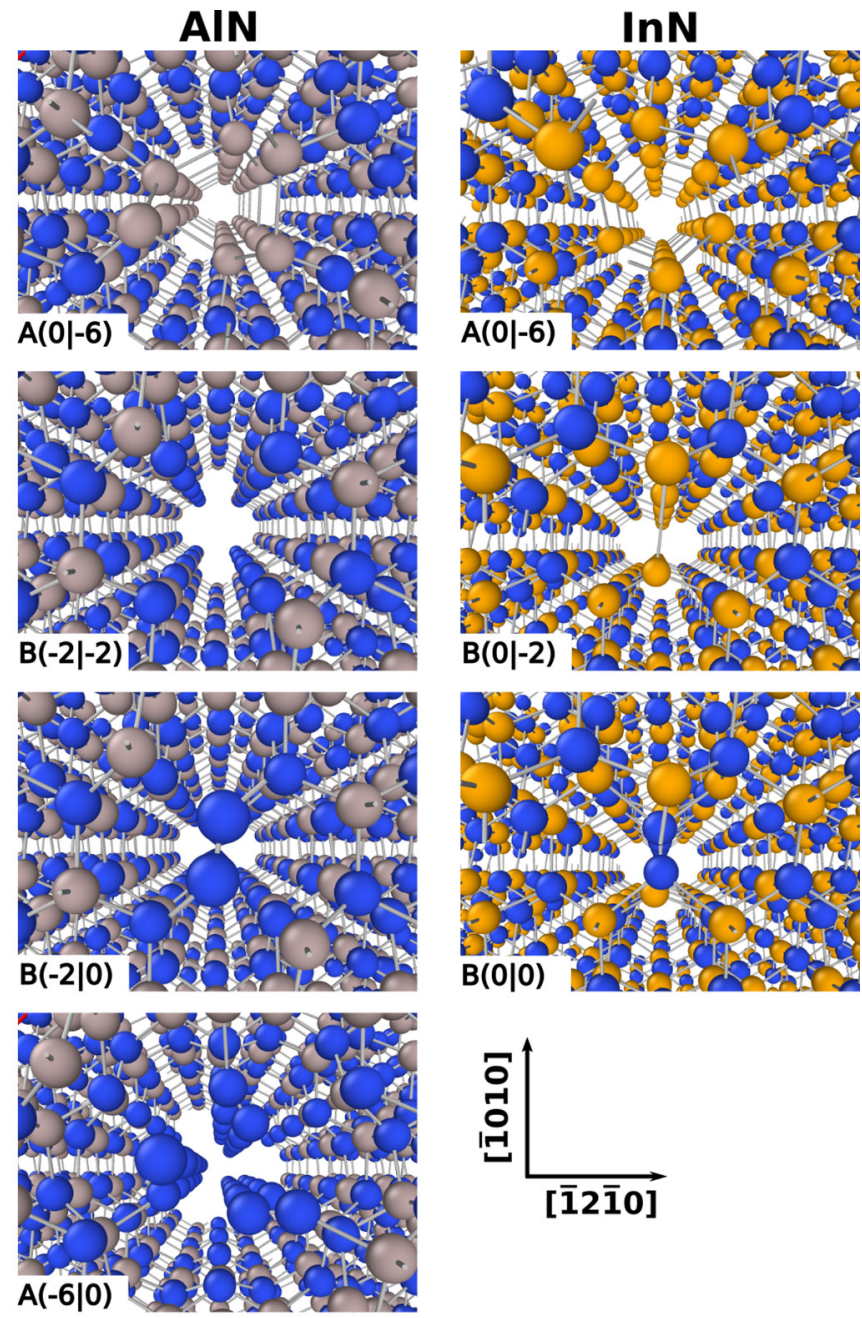

FIG. 4. Perspective view of the most stable dislocation core configurations for AlN (left) and InN (right), relaxed by first-principles calculations. Nitrogen, aluminum, and indium atoms are represented by blue, gray, and orange spheres, respectively.

$2.73 \AA$ and $2.83 \AA$, thus close to the first neighbors distance in bulk aluminum $(2.86 \AA)$.

The second low energy configuration, $\mathrm{B}(-2 \mid-2)$, is characterized by a core free of central $\mathrm{N}$ and $\mathrm{Al}$ atoms. Since the dislocation is centered on a bond, the void volume is smaller than in other open core configurations like $\mathrm{A}(-6 \mid-6)$ (Figs. 1 and 4). The analysis of the relaxed structure reveals the presence of four columns of Al-N dimers along $\hat{\mathbf{z}}$. These dimer bonds strengthen when core atoms are removed, according to a mechanism that is well known for nonpolar prismatic nitride surfaces [54,55], and also occurs for the $A(-6 \mid-6)$ core in $\mathrm{GaN}$ [5]. These dimers are characterized by a length equal to $1.83 \AA$, i.e., $4 \%$ lower than the bulk $\mathrm{Al}-\mathrm{N}$ distance. They are also slightly buckled, because the $\mathrm{Al}$ atoms are moving closer to the inner surfaces than the $\mathrm{N}$ atoms. Previous studies on $\mathrm{GaN}$ concluded that this atomic organization allows for a $s p^{2}\left(s p^{3}\right)$ character of $\mathrm{Al}(\mathrm{N})$ atoms-related states [5,55].

The $\mathrm{B}(-2 \mid 0)$ relaxed structure shares several characteristics with the previously discussed $\mathrm{B}(-2 \mid-2)$ configuration. In fact, the same dimer strengthening mechanism occurs, leading to 
four lines of buckled dimers along $\hat{\mathbf{z}}$ (Fig. 4). However, the core now contains $\mathrm{N}-\mathrm{N}$ dimers. The dimer length is $1.18 \AA$, that is larger than for an isolated $\mathrm{N}_{2}$ molecule with a triple bond $(1.11 \AA)$, but also lower than the double bond length $(1.25 \AA)$. The N-N dimer is tilted by $0.19 \AA$ along $\hat{\mathbf{x}}$, but not along $\hat{\mathbf{z}}$. Furthermore, using a distance criterion only, each nitrogen atom seems to be bonded to one $\mathrm{Al}$ atom on the inner core surfaces, with a bond length of $2.10 \AA$. These aluminum atoms are slightly less close to the inner surfaces than the other $\mathrm{Al}$ atoms, supporting the idea of a bond with the $\mathrm{N}_{2}$ dimer.

Finally, the last AIN core configuration with low energy is $\mathrm{A}(-6 \mid 0)$ (Fig. 4). It can be viewed as an open core containing $\mathrm{N}_{2}$ dimers. The open core geometry is similar to the one reported for $\mathrm{GaN}$, i.e., inner surface $\mathrm{Al}$ and $\mathrm{N}$ atoms form dimers oriented along $\hat{\mathbf{z}}$ [5]. The dimer length is $1.80 \AA$ and it is tilted because $\mathrm{Al}$ atoms are closer to inner surfaces than $\mathrm{N}$ atoms. Unlike $\mathrm{B}(-2 \mid-2)$ and $\mathrm{B}(-2 \mid 0)$, the dislocation is centered on one hexagon, leading to six columns of Al-N dimers on the core inner surfaces. In the core center, we observed the formation of three $\mathrm{N}_{2}$ dimers per Burgers vector during the relaxation. The dimer length is $1.11 \AA$, thus suggesting the presence of a triple bond between nitrogen atoms. Compared to the $\mathrm{N}_{2}$ dimers in $\mathrm{B}(-2 \mid 0)$, a significant tilting along $\hat{\mathbf{z}}$ is obtained, with a height difference of $0.4 \AA$ between the two $\mathrm{N}$ atoms. Each dimer seem to be connected to the core inner surfaces thanks to a bond with an $\mathrm{Al}$ atom, of length equal to $2.1 \AA$. The core inner surface dimerization is only weakly perturbed by the presence of these $\mathrm{N}_{2}$ dimers.

Now we describe the three relaxed InN low energy core configurations. The $\mathrm{A}(0 \mid-6)$ core is weakly modified during force relaxation, and the final geometry, shown in Fig. 4, is different from $\mathrm{AlN} A(0 \mid-6)$. In fact, there is now only one kind of In-In bond in the core, with a length of $2.84 \AA$. Then the $\operatorname{InN} \mathrm{A}(0 \mid-6)$ core can still be viewed as two interwoven helical chains of InN atoms along the $\hat{\mathbf{z}}$ axis, each one having a period of $2 c_{0}$. But there are no zigzag dimer chains along $\hat{\mathbf{z}}$, as for AlN. Comparing the In-In bond length with the first neighbor distance in bulk indium $(3.25 \AA)$ suggests that this indium core substructure is compressed into the dislocation core.

The second stable InN configuration is $\mathrm{B}(0 \mid-2)$ (Fig. 4). It features an In-In dimer in the dislocation core, with a bond length of $2.69 \AA$. The two In atoms have the same position along $\hat{\mathbf{z}}$, but not along $\hat{\mathbf{x}}$ with a difference of $0.3 \AA$. Each In atom is bonded to two $\mathrm{N}$ atoms on the core inner surfaces, with bond lengths of 2.20 and $2.22 \AA$, i.e., close to the In-N bond length in bulk InN (2.18 $\AA$ ). In addition, each In atom is also bonded to two inner surface In atoms, with bond lengths of $2.77 \AA$ and $3.03 \AA$. The angles associated with these five bonds range from $85^{\circ}$ to $118^{\circ}$. At last, we found that the presence of the central In atoms prevents the strengthening of In-N dimers on the core inner surfaces, their bond length of $2.16 \AA$ remaining close to the In-N distance in InN bulk.

Finally, we focus on the $\mathrm{InN} \mathrm{B}(0 \mid 0)$ relaxed configuration. Figure 4 reveals that the core is composed of In-In and N-N dimers alternating along the dislocation line. The In-In dimer can be compared to the central In-In dimer in the $\mathrm{B}(0 \mid-2)$ configuration. The bond length is $2.70 \AA$, with no tilts along both $\hat{\mathbf{z}}$ and $\hat{\mathbf{x}}$. Like in $\mathrm{B}(0 \mid-2)$, each of these In atoms appears to be further bonded to two $\mathrm{N}$ atoms, with bond lengths $2.19 \AA$ and $2.21 \AA$, and to two In atoms, with bond lengths $2.87 \AA$ and $2.99 \AA$. The central N-N dimer is characterized by a length of $1.26 \AA$, suggesting a double bond. As for In-In dimer, each of these $\mathrm{N}$ atoms appears to be connected to two In atoms on core inner surfaces, with bond lengths of $2.32 \AA$ and $2.36 \AA$, thus slightly stretched compared to the In-N bulk bond length.

\section{ELECTRONIC STRUCTURE}

Electronic structure calculations have been studied using calculations in the small supercell. For these analyses, the issue lies in the alignment of electronic levels that must be performed to have a common reference between dislocation and bulk calculations. Two methods are commonly used. A first one requires one to compute the average electrostatic potentials to determine the relative shift. However, for the defected system, the average should be computed in a weakly perturbed region.

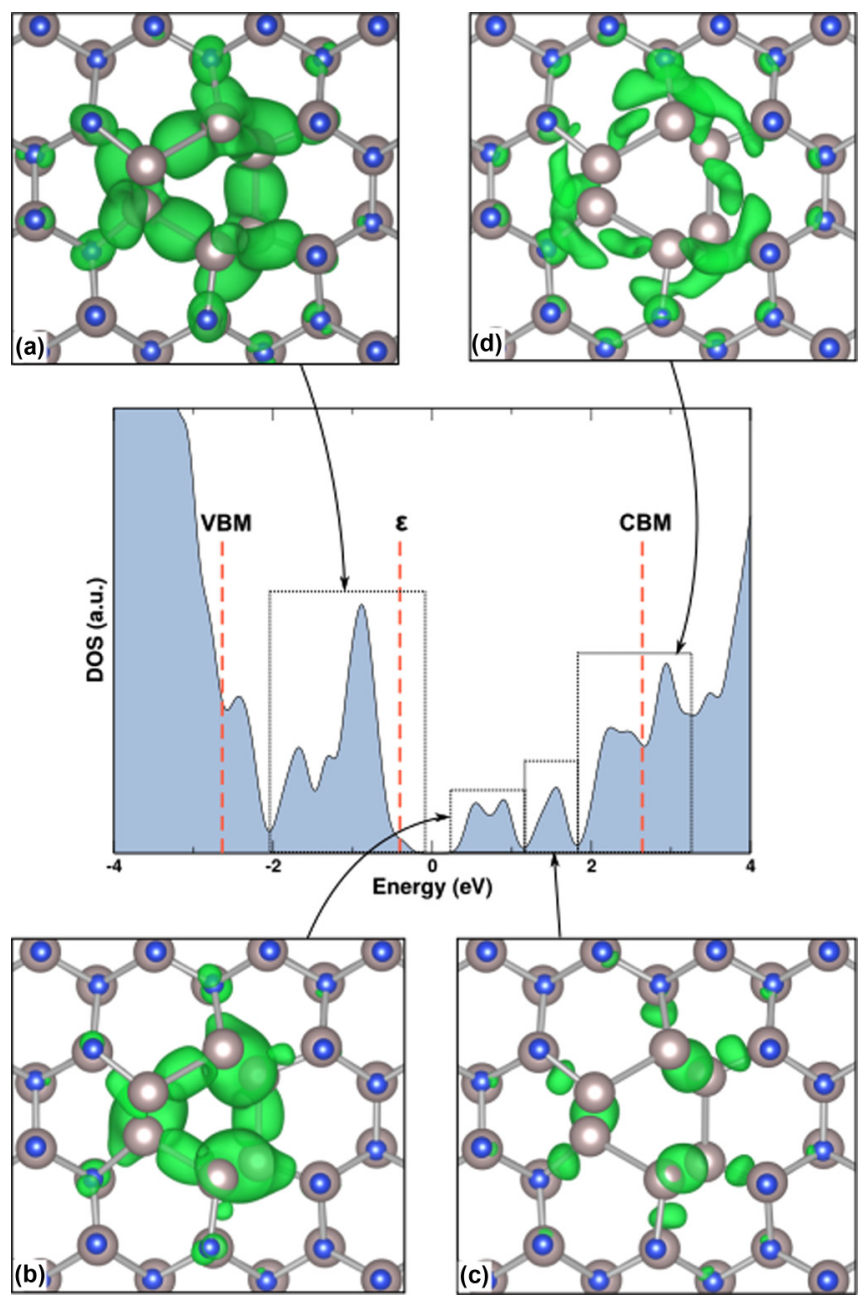

FIG. 5. Density of states for the $\mathrm{A}(0 \mid-6)$ dislocation core in AlN, showing the highest occupied electronic level $(\epsilon)$, as well as the valence band maximum (VBM) and conduction band minimum (CBM) of perfect bulk, after alignment. The additional figures (a)-(d) display isosurface representations of the local DOS, integrated over the energy range corresponding to the dashed frames. Bulk Fermi level is set to $0 \mathrm{eV}$. 
In the case of defects like dislocations, inducing a long range stress field around the core, such a region might be difficult to find. A second approach consists in determining the shift by comparing the lowest energy states, which are presumably not perturbed by the presence of the defect. In the case of AlN, both methods have been used, yielding similar results. However, no good agreement was obtained for InN. In particular, we found significant differences in average electrostatic potentials depending on the selected region. Thus, for $\mathrm{InN}$, the electronic structure of dislocation cores was aligned on bulk electronic structure by comparing low energy " $d$ " states.

We first consider the case of the most stable dislocation cores in AlN, starting with the $\mathrm{A}(0 \mid-6)$ configuration. Figure 5 shows the computed density of states for this core structure, revealing the presence of several states in the band gap. A first group of occupied states with energies ranging approximately from $-2 \mathrm{eV}$ to $-0.2 \mathrm{eV}$ corresponds mostly to $\mathrm{Al}-\mathrm{Al}$ bonds in the dislocation core, with a small contribution from states associated to first neighbors nitrogen atoms [Fig. 5(a)]. Regarding empty states, a first group, from $0.2 \mathrm{eV}$ to $1.2 \mathrm{eV}$, corresponds to $\mathrm{Al}-\mathrm{Al}$ antibonding states [Fig. 5(b)]. The next one, displayed in Fig. 5(c), is associated to both core Al-Al bonds and $\mathrm{Al}-\mathrm{N}$ bonds in the core. Finally, states with energies in the range of $\mathrm{CBM}$ appear to be related to core $\mathrm{Al}$ atoms, and also to $\mathrm{N}$ atoms, first neighbors of the dislocation [Fig. 5(d)]. The large band gap of AlN is then almost completely filled with dislocation core states, mostly associated to Al-Al bonds, which are characterized by a strong dispersion

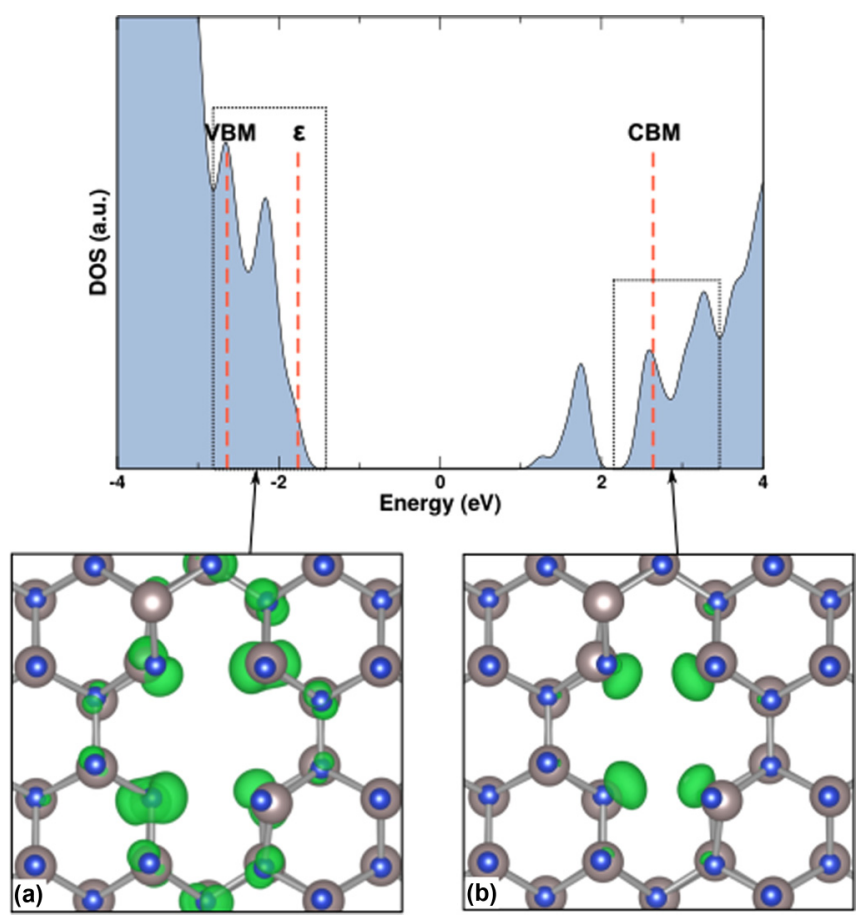

FIG. 6. Density of states for the $\mathrm{B}(-2 \mid-2)$ dislocation core in AlN, showing the highest occupied electronic level $(\epsilon)$, as well as the valence band maximum (VBM) and conduction band minimum (CBM) of perfect bulk, after alignment. The additional figures (a) and (b) display isosurface representations of the local DOS, integrated over the energy range corresponding to the dashed frames. Bulk Fermi level is set to $0 \mathrm{eV}$. along the dislocation line. This result indicates a metallic behavior along the dislocation line, a detrimental property for nitride-based devices. Note that the presence of the metallic states is not surprising since the distances between $\mathrm{Al}$ atoms in the dislocation core are similar to those in bulk aluminum. A similar conclusion was already drawn by Northrup for the same dislocation core configuration in GaN [21].

The electronic structure of the second configuration of interest for AlN, B(-2|-2), is shown in Fig. 6. Unlike the previous one, a large part of the original band gap remains empty of states. Dislocation occupied states are relatively close to the VBM, and are localized mainly on $\mathrm{N}$ atoms surrounding the empty center, seemingly with a " $p$ " character [Fig. 6(a)]. A well defined peak at about $1.8 \mathrm{eV}$, i.e., $1 \mathrm{eV}$ below the CBM, corresponds to empty " $p$ " states on low coordinated nitrogen and aluminum atoms. Both these occupied and unoccupied states are probably equivalent to prismatic surface states, forming the interior of the open $\mathrm{B}(-2 \mid-2)$ core. This equivalence has already been proposed in the case of $\mathrm{GaN}$ and the open $A(-6 \mid-6)$ dislocation core [5]. Compared to a prismatic surface, the shallow character of the states is due to the distortion arising from the dislocation. We also found a second peak centered on the CBM, represented in Fig. 6(b), exhibiting the same properties as the peak at $1.8 \mathrm{eV}$. Matsubara and co-workers determined the same behavior for the $\mathrm{B}(-2 \mid-2)$ core in $\mathrm{GaN}$, and demonstrated that these two peaks correspond to the same ensemble of states, artificially splitted in two by the large strain due to the short distance between dislocations in

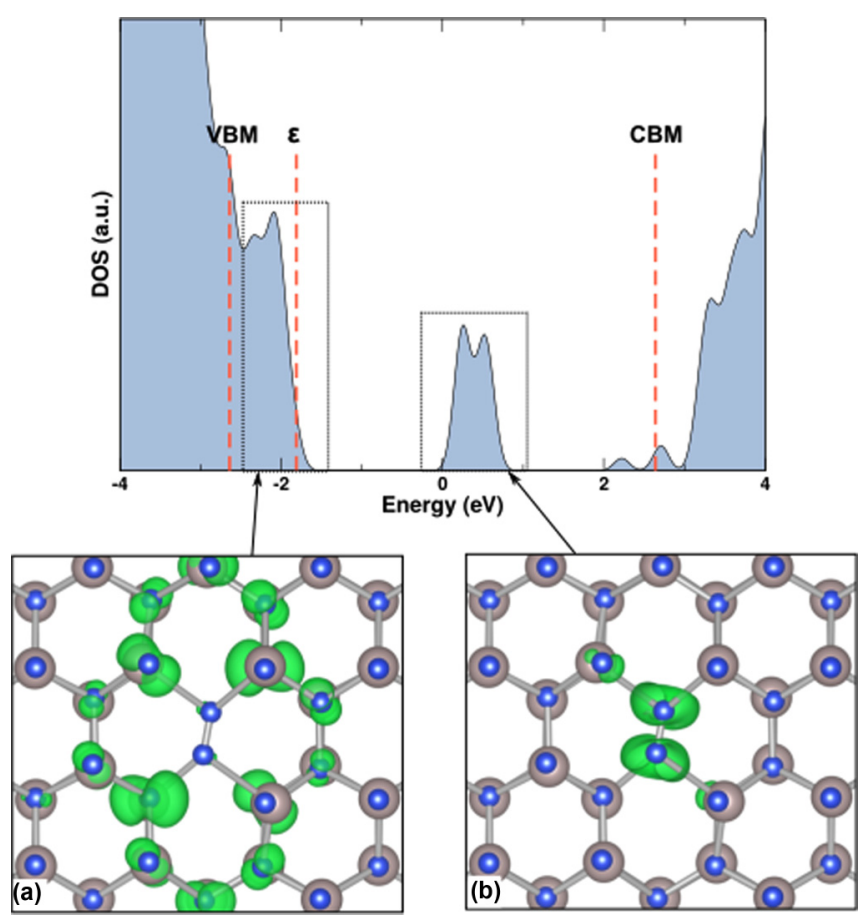

FIG. 7. Density of states for the $\mathrm{B}(-2 \mid 0)$ dislocation core in AlN, showing the highest occupied electronic level $(\epsilon)$, as well as the valence band maximum (VBM) and conduction band minimum (CBM) of perfect bulk, after alignment. The additional figures (a) and (b) display isosurface representations of the local DOS, integrated over the energy range corresponding to the dashed frames. Bulk Fermi level is set to $0 \mathrm{eV}$. 
the supercell [23]. We can then conclude that, for $\mathrm{B}(-2 \mid-2)$, all electronic states associated with the dislocation core are shallow levels, with little influence on electronic properties.

For the $\mathrm{B}(-2 \mid 0)$ core geometry, a first group of states with energies just above the VBM is identified. These states bear a resemblance with those observed at equivalent energies for the $\mathrm{B}(-2 \mid-2)$ core, although they appear to be more delocalized in the vicinity of the core [Fig. 7(a)]. These states are mostly localized on nitrogen atoms, first and second neighbors of the dislocation core. Two splitted small peaks are also present close to the CBM, with a similar localization, as for $\mathrm{B}(-2 \mid-2)$. Now, unlike $\mathrm{B}(-2 \mid-2)$, the presence of the central $\mathrm{N}-\mathrm{N}$ dimers induces unoccupied states for energies ranging from 0 to $1 \mathrm{eV}$ [Fig. 7(b)]. Those latter are located on the core nitrogen atoms, and exhibit a clear $\pi^{*}$ character. Such antibonding orbitals are in agreement with the analysis made in the previous section, concluding to the presence of a double bond in the $\mathrm{N}-\mathrm{N}$ dimer.

The DOS for the last stable AIN screw core configuration is shown in Fig. 8. For this core, shallow occupied states appear

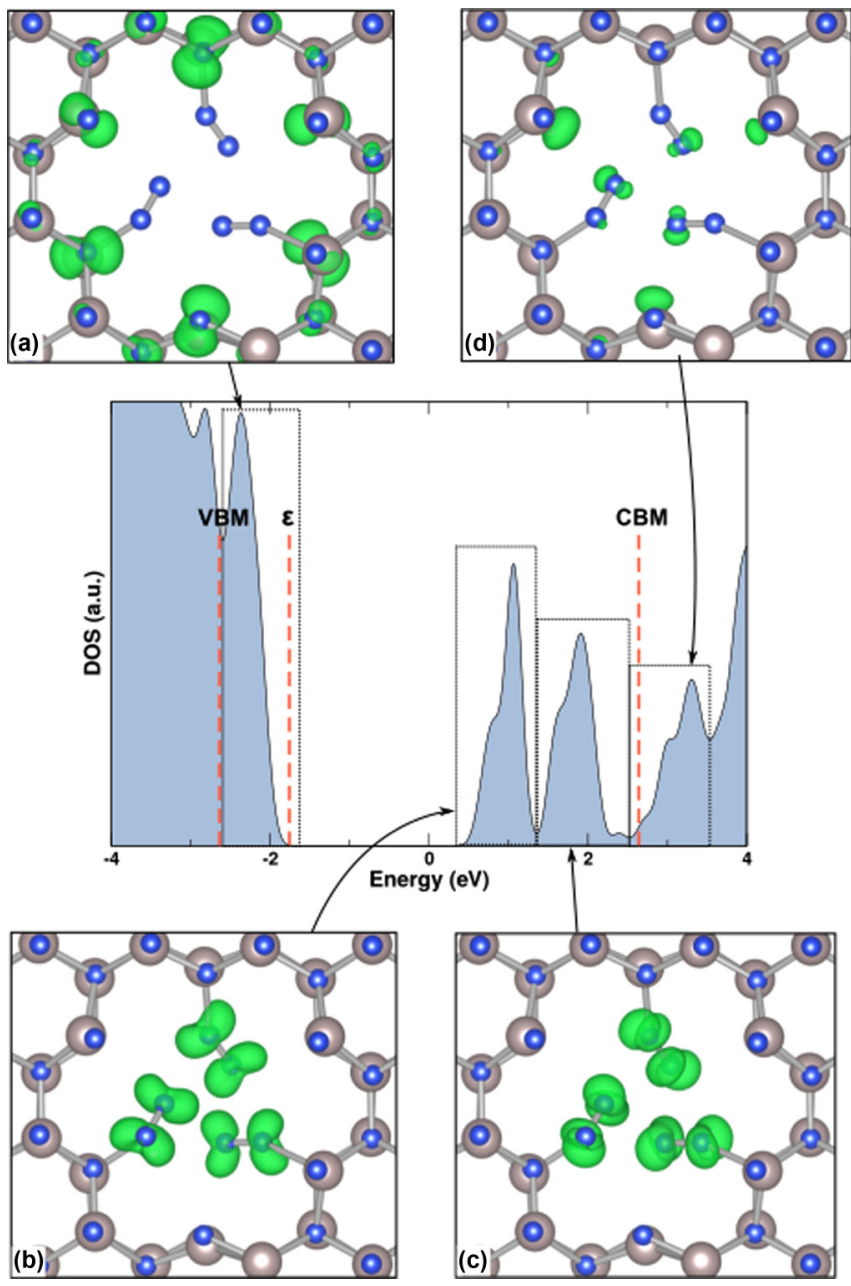

FIG. 8. Density of states for the $\mathrm{A}(-6 \mid 0)$ dislocation core in AlN, showing the highest occupied electronic level $(\epsilon)$, as well as the valence band maximum (VBM) and conduction band minimum (CBM) of perfect bulk, after alignment. The additional figures (a)-(d) display isosurface representations of the local DOS, integrated over the energy range corresponding to the dashed frames. Bulk Fermi level is set to $0 \mathrm{eV}$. located on noncentral $\mathrm{N}$ atoms, which are characterized by a lower coordination than in bulk [Fig. 8(a)]. These states are similar to those obtained for the "open" $A(-6 \mid-6)$ core. The salient feature of the $\mathrm{B}(-6 \mid 0)$ dislocation core is the presence of empty levels, clearly identified as $\pi *$ molecular orbitals, deep into the gap (two peaks at approximately $1 \mathrm{eV}$ and $2 \mathrm{eV}$ ) [Figs. 8(b) and 8(c)]. Finally, the analysis of the peak located just above the CBM reveals empty p states on (i) $\mathrm{N}$ atoms at the apparent loose end of the $\mathrm{N}-\mathrm{N}$ dimer and (ii) undercoordinated atoms forming the interior of the dislocation cores [Fig. 8(d)].

In the previous section, we tentatively conclude that the central N-N dimers are in fact close to $\mathrm{N}_{2}$ molecules. To confirm this point, we analyzed the electronic structure of an isolated $\mathrm{N}_{2}$ molecule. It shows that the maximum charge density between the two $\mathrm{N}$ atoms is $4.93 e^{-} \AA^{-3}$. In the case of the $\mathrm{N}-\mathrm{N}$ dimer in the dislocation core, the maximum charge density is found to be very close, equal to $4.79 e^{-} \AA^{-3}$, which confirms our point. We also discussed the presence of a bond between this $\mathrm{N}-\mathrm{N}$ dimer and an aluminum atom, with a separation slightly larger than the $\mathrm{Al}-\mathrm{N}$ bond in bulk AlN. Comparing the maximum charge density in bulk AlN (3.17 $\left.e^{-} \AA^{-3}\right)$ and for this bond $\left(3.64 e^{-} \AA^{-3}\right)$ indicates that there is a real ionocovalent bond between $\mathrm{Al}$ and the $\mathrm{N}_{2}$ molecule in the dislocation core.

We now turn to indium nitride, and first to the $\mathrm{A}(0 \mid-6)$ configuration shown in Fig. 9. The electronic gap is

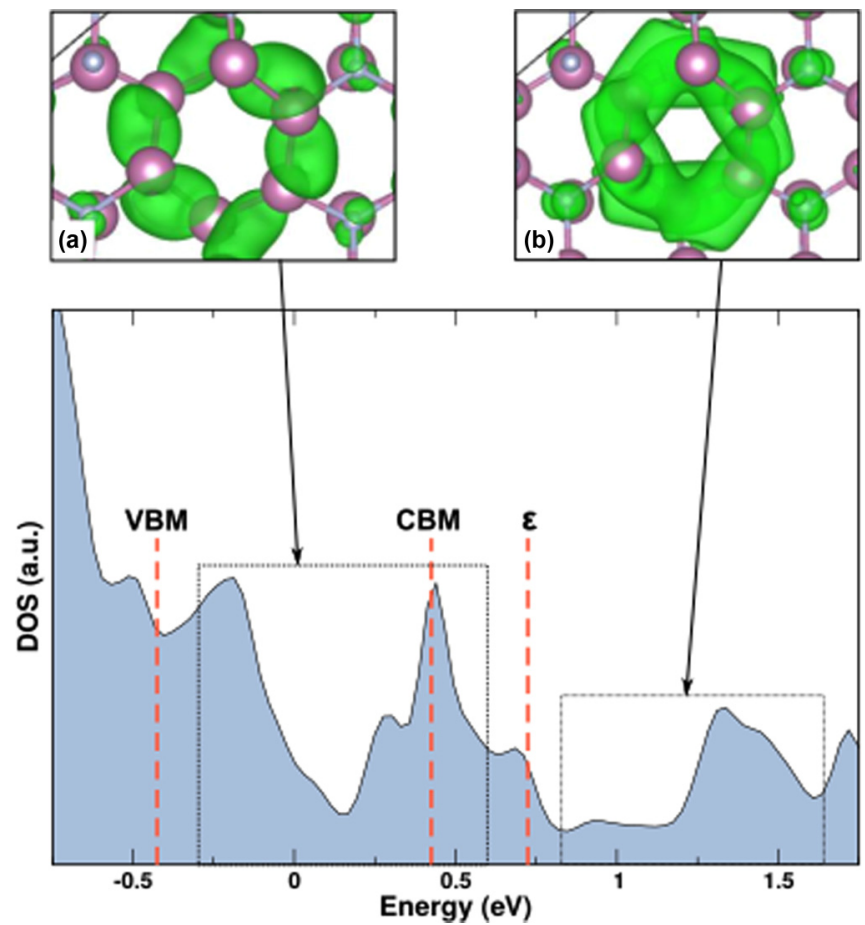

FIG. 9. Density of states for the $\mathrm{A}(0 \mid-6)$ dislocation core in InN, showing the highest occupied electronic level $(\epsilon)$, as well as the valence band maximum (VBM) and conduction band minimum (CBM) of perfect bulk, after alignment. The additional figures (a) and (b) display isosurface representations of the local DOS, integrated over the energy range corresponding to the dashed frames. Bulk Fermi level is set to $0 \mathrm{eV}$. 


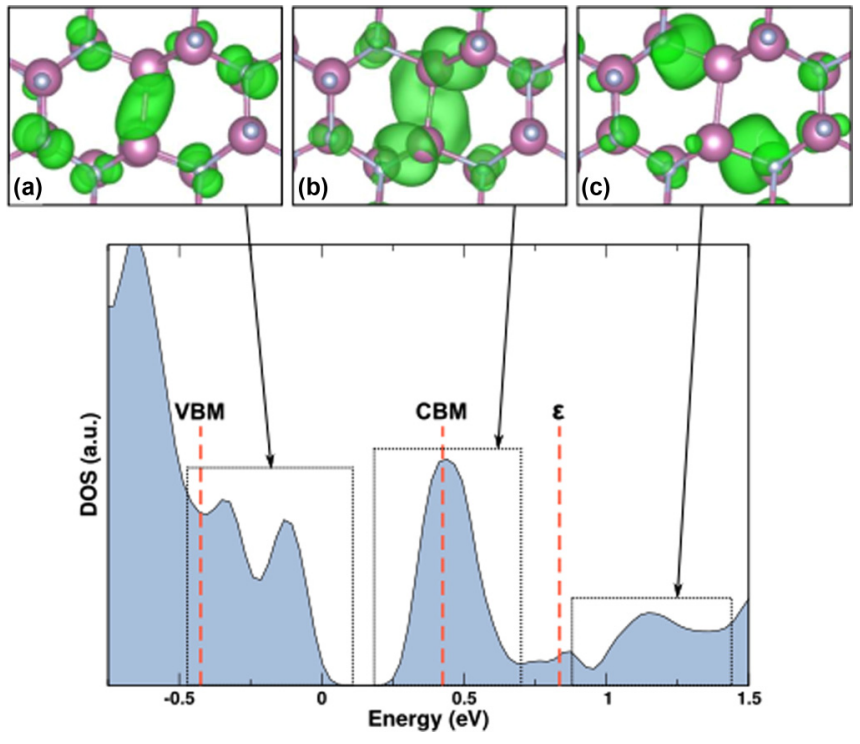

FIG. 10. Density of states for the $\mathrm{B}(0 \mid-2)$ dislocation core in InN, showing the highest occupied electronic level $(\epsilon)$, as well as the valence band maximum (VBM) and conduction band minimum (CBM) of perfect bulk, after alignment. The additional figures (a)-(c) display isosurface representations of the local DOS, integrated over the energy range corresponding to the dashed frames. Bulk Fermi level is set to $0 \mathrm{eV}$.

completely filled with states originating from the dislocation core. Those are $\sigma$ bonding states localized on the central indium atoms [Fig. 9(a)]. Another related group of electronic states, associated to energies from $0.8 \mathrm{eV}$ to $1.6 \mathrm{eV}$, corresponds to $\pi^{*}$ antibonding orbitals, also localized on central In atoms [Fig. 9(b)]. Like for AlN and GaN, these states suggest a metallic behavior along the dislocation line. Furthermore, it is noteworthy that the highest occupied level has a higher energy than the CBM, a feature which is also found for threading edge dislocations in $\mathrm{InN}$ and attributed to undercoordinated In atoms $[15,16]$. It implies that the dislocation will supply electron carriers to the conduction band of InN.

The second stable core in $\operatorname{InN}, \mathrm{B}(0 \mid-2)$, shares the same property, i.e., its highest occupied level has an energy above the CBM. We found a first group of shallow levels, until about $0.5 \mathrm{eV}$ above the VBM (Fig. 10). They correspond to both p-like orbitals localized on nitrogen first neighbors of the dislocation core center and $\sigma$ orbitals localized on In-In central bonds [Fig. 10(a)]. At higher energies, there is a peak centered on the CBM, associated mainly with bonding states involving the central In atoms [Fig. 10(b)]. Finally, our analysis reveals unoccupied levels associated with the dislocation core, for energies ranging from $0.85 \mathrm{eV}$ to $1.45 \mathrm{eV}$ in Fig. 10 . These resonant states are originating from dangling bonds on In atoms, first neighbors of the central In atoms [Fig. 10(c)]. States localized on nitrogen atoms with $s$ - and $p$-like character also apparently contribute to these levels.

At last, Fig. 11 shows the electronic structure for the $\mathrm{B}(0 \mid 0)$ dislocation core in $\mathrm{InN}$. The density of states is characterized by several merged peaks, from $-0.35 \mathrm{eV}$ to $0.25 \mathrm{eV}$. The corresponding states seem to be rather similar to those

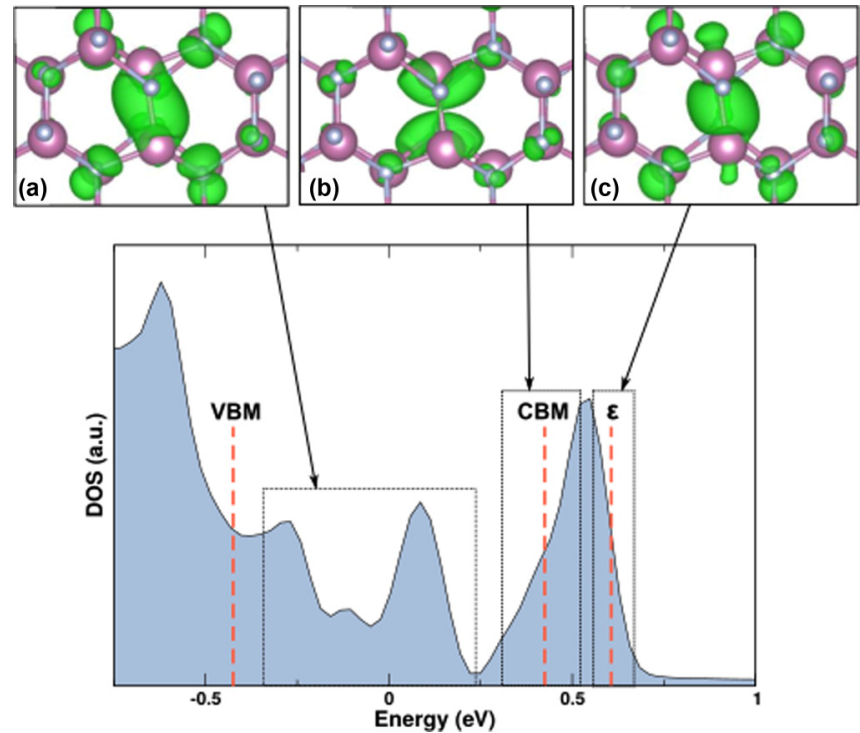

FIG. 11. Density of states for the $\mathrm{B}(0 \mid 0)$ dislocation core in $\mathrm{InN}$, showing the highest occupied electronic level $(\epsilon)$, as well as the valence band maximum (VBM) and conduction band minimum (CBM) of perfect bulk, after alignment. The additional figures (a)-(c) display isosurface representations of the local DOS, integrated over the energy range corresponding to the dashed frames. Bulk Fermi level is set to $0 \mathrm{eV}$.

identified for slightly lower energies and the $\mathrm{B}(0 \mid-2)$ core [Figs. 10(a) and 11(a)]. However, the dispersion is stronger, and as a result, the InN bulk band gap is fully filled with dislocation-related states. One then may expect a metalliclike behavior along the dislocation line. Furthermore, the large peak in the range $0.25-0.7 \mathrm{eV}$ is different to the one centered at $\mathrm{CBM}$ for the $\mathrm{B}(0 \mid-2)$ core. In fact, the left part of the peak, centered on CBM, is associated to $\pi$-like states localized on the central N-N dimers [Figs. 11(b)]. The right part of the peak appears to correspond to $\sigma$-like states from the In-In dimers [Figs. 11(c)]. As for $\mathrm{A}(0 \mid-6)$ and $\mathrm{B}(0 \mid-2)$, we found that the highest occupied level has an energy above the CBM.

\section{DISCUSSION}

Our first-principles calculations revealed several original stable configurations for the prismatic screw dislocation in AlN and $\mathrm{InN}$, increasing our knowledge for these materials to the same level as for gallium nitride. We now aim at comparing screw dislocations for all three materials and tentatively explaining the sequence of stable dislocation cores. For GaN, we refer to the results from the work of Matsubara et al. [23], which have been obtained in a similar theoretical framework as ours.

The general properties of $\mathrm{AlN}, \mathrm{GaN}$, and $\mathrm{InN}$ are well known [56]. They are characterized by a strong ionic character, like II-VI materials, and exhibit a mechanical behavior similar to IV-IV ceramic systems like diamond or SiC. Concerning ionicity, AlN is characterized by the highest value, while GaN and $\mathrm{InN}$ are alike. One may then expect significant charge transfers into the dislocation core, especially for nonstoichiometric 


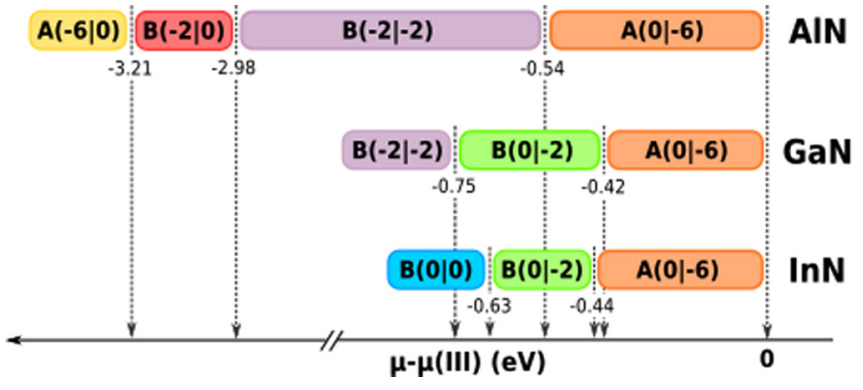

FIG. 12. Schematic view of the most stable prismatic screw configurations in $\mathrm{AlN}, \mathrm{GaN}$, and $\mathrm{InN}$, as a function of the chemical potential.

configuration. Elastic constants are close and very high for $\mathrm{AlN}$ and GaN, whereas InN is softer. Overall, bonds in III-N systems are strong and rigid, and reconstruction mechanisms in the core, lowering strain energy, can be anticipated. At last, the electronic band gap is very high for AlN, intermediate for $\mathrm{GaN}$, and small for $\mathrm{InN}$.

Despite these differences, it is remarkable that the same configuration is found for a prismatic screw dislocation in all three materials for III-rich conditions (Fig. 12). In fact, our calculations undoubtedly show that the $\mathrm{A}(0 \mid-6)$ core is the most stable one, like in GaN [20]. In the A family, the screw dislocation is centered on one hexagon formed by alternating III and $\mathrm{N}$ atoms. Removing these atoms greatly relieves the large shear strains in the dislocation core. This is especially important for III-N materials like AlN and GaN with rigid ionocovalent bonds, as explained in the previous paragraph. In all three materials, $\mathrm{A}(0 \mid-6)$ can be obtained by removing $\mathrm{N}$ atoms while leaving III-type atoms. For AIN and $\mathrm{GaN}$, the significant displacement of $\mathrm{Al}$ and $\mathrm{Ga}$ atoms during relaxation suggests that an optimal configuration is obtained. However, we do not observe a similar phenomenon for In atoms during the relaxation of the $\mathrm{A}(0 \mid-6)$ core in InN. One then cannot exclude that a lower energy configuration might be obtained by slightly decreasing the number of In atoms in the core. For all three materials, the $\mathrm{A}(0 \mid-6)$ core configuration possesses a metalliclike electronic structure where almost no gap is present. The defect related unoccupied energy levels are associated with delocalized states running, as helices, along metal atoms belonging to the six-atoms ring [3]. This extended metallization gives rise to conduction channels which allow the screw dislocations to provide pathways to leakage currents in III-N based electronic devices.

Now progressing towards N-rich conditions, we observe a change of the most stable core for approximately the same chemical potential value for $\mathrm{GaN}$ and $\mathrm{InN},-0.42 \mathrm{eV}$ and $-0.44 \mathrm{eV}$, respectively. The $\mathrm{B}(0 \mid-2)$ geometry can also be viewed as an open core subsequently filled with III atoms, but it is now centered on a bond (B family). For AlN, its energy is slightly too high to be stable; otherwise it would appear for approximately the same chemical potential value (Fig. 2), again marking the similarities between all three III-N materials.

The next most stable configuration for AlN and GaN, $\mathrm{B}(-2 \mid-2)$, is like the previous one, but with the central III atoms removed. Such an open core allows for a preserved gap, with only shallow levels. Interestingly, it corresponds to a high energy in InN, for which the full bond-centered core $\mathrm{B}(0 \mid 0)$ is predicted instead. It seems that, in the case of $\mathrm{InN}$, the distortion energy gain obtained by removing central atoms is not compensated by the energy cost associated to the core internal surface creations. It can probably be explained by the relative softness of InN compared to AIN and GaN.

Finally, we found two stable nitrogen filled dislocation cores for AlN in $\mathrm{N}$-rich conditions, one centered on a bond $[\mathrm{B}(-2 \mid 0)]$ and the other centered on a hexagon $[\mathrm{A}(-6 \mid 0)]$. They appear as open structures with enough space to accommodate nitrogen dimers. Those are in fact nitrogen molecules either with a double bond between the $\mathrm{N}$ atoms and bonded to two $\mathrm{Al}$ atoms $[\mathrm{B}(-2 \mid 0)]$ or with a triple bond and bonded to a single $\mathrm{Al}[\mathrm{A}(-6 \mid 0)]$. These electronic configurations are possible because of the high ionicity of AlN, with nitrogen atoms easily capturing electrons from aluminum atoms. The presence of these $\mathrm{N}_{2}$ molecules leads to deep levels in the electronic gap, suggesting that screw dislocations in these configurations should act as radiative recombination centers. These two configurations are obtained for very low values of chemical potentials, only possible in AlN because of its high formation energy. It is then highly unlikely that they could exist in $\mathrm{GaN}$ and InN.

\section{CONCLUSIONS}

We have performed a comprehensive study of the threading screw dislocation in $\mathrm{AlN}$ and $\mathrm{InN}$, using first-principles calculations, two materials for which we had little knowledge of this extended defect. Taking into account insights gained for $\mathrm{GaN}$, a large number of possible configurations was tried, with a variable stoichiometry. These calculations allowed for determining the most stable dislocation cores for different experimental growth conditions. For both AlN and $\mathrm{InN}$, new configurations are predicted compared to the current state of the art. An Al-filled (In-filled) dislocation core involving a metalliclike behavior along the dislocation line is predicted in Al-rich (In-rich) conditions in AIN ( InN). Other stable dislocation cores in $\mathrm{InN}$ are characterized by the partial or complete closure of the gap and the injection of electrons into the bulk conduction band. Finally, we report two original dislocation geometries for AlN in N-rich conditions, in which the core is seemingly filled with nitrogen molecules. Electronic structure analysis revealed that these two core geometries introduce deep levels in the electronic gap.

Future investigations should be aimed at determining the influence of dopants or other impurities on the properties of these dislocations. Another interesting research direction could be ternary alloys like InGaN and AlGaN, two materials with great prospect of applications.

\section{ACKNOWLEDGMENTS}

The computations were performed at "CRIANN," Centre Régional Informatique et d'Applications Numériques de Normandie, http://www.criann.fr, and at the Mesocentre SPIN at the University of Poitiers. 
[1] T. D. Moustakas and R. Paiella, Rep. Prog. Phys. 80, 106501 (2017).

[2] J. W. P. Hsu, M. J. Manfra, R. J. Molnar, B. Heying, and J. S. Speck, Appl. Phys. Lett. 81, 79 (2002).

[3] I. Belabbas, J. Chen, and G. Nouet, Comput. Mater. Sci. 90, 71 (2014).

[4] S. Usami, Y. Ando, A. Tanaka, K. Nagamatsu, M. Deki, M. Kushimoto, S. Nitta, Y. Honda, H. Amano, Y. Sugawara, Y.-Z. Yao, and Y. Ishikawa, Appl. Phys. Lett. 112, 182106 (2018).

[5] J. Elsner, R. Jones, P. K. Sitch, V. D. Porezag, M. Elstner, T. Frauenheim, M. I. Heggie, S. Öberg, and P. R. Briddon, Phys. Rev. Lett. 79, 3672 (1997).

[6] I. Belabbas, P. Ruterana, J. Chen, and G. Nouet, Philos. Mag. 86, 2241 (2006).

[7] A. F. Wright and U. Grossner, Appl. Phys. Lett. 73, 2751 (1998).

[8] J. Elsner, R. Jones, M. I. Heggie, P. K. Sitch, M. Haugk, T. Frauenheim, S. Öberg, and P. R. Briddon, Phys. Rev. B 58, 12571 (1998).

[9] S. M. Lee, M. A. Belkhir, X. Y. Zhu, Y. H. Lee, Y. G. Hwang, and T. Frauenheim, Phys. Rev. B 61, 16033 (2000).

[10] A. Béré and A. Serra, Phys. Rev. B 65, 205323 (2002).

[11] C. J. Fall, R. Jones, P. R. Briddon, A. T. Blumenau, T. Frauenheim, and M. I. Heggie, Phys. Rev. B 65, 245304 (2002).

[12] L. Lymperakis, J. Neugebauer, M. Albrecht, T. Remmele, and H. P. Strunk, Phys. Rev. Lett. 93, 196401 (2004).

[13] S. K. Rhode, M. K. Horton, M. J. Kappers, S. Zhang, C. J. Humphreys, R. O. Dusane, S. L. Sahonta, and M. A. Moram, Phys. Rev. Lett. 111, 025502 (2013).

[14] A. F. Wright and J. Furthmüller, Appl. Phys. Lett. 72, 3467 (1998).

[15] Y. Takei and T. Nakayama, J. Cryst. Growth 311, 2767 (2009).

[16] E. Kalesaki, J. Kioseoglou, L. Lymperakis, P. Komninou, and T. Karakostas, Appl. Phys. Lett. 98, 072103 (2011).

[17] T. Akiyama, R. Sakaguchi, K. Nakamura, and T. Ito, Phys. Status Solidi B 254, 1600694 (2017).

[18] M. Funato, R. G. Banal, and Y. Kawakami, AIP Adv. 5, 117115 (2015).

[19] A. T. Blumenau, J. Elsner, R. Jones, M. I. Heggie, S. Öberg, T. Frauenheim, and P. R. Briddon, J. Phys.: Condens. Matter 12, 10223 (2000).

[20] J. E. Northrup, Appl. Phys. Lett. 78, 2288 (2001).

[21] J. E. Northrup, Phys. Rev. B 66, 045204 (2002).

[22] I. Belabbas, J. Chen, and G. Nouet, Comput. Mater. Sci. 51, 206 (2011).

[23] M. Matsubara, J. Godet, L. Pizzagalli, and E. Bellotti, Appl. Phys. Lett. 103, 262107 (2013).

[24] M. Matsubara, L. Pizzagalli, and E. Bellotti, Phys. Status Solidi C 11, 521 (2014).

[25] J. Kioseoglou, E. Kalesaki, I. Belabbas, J. Chen, G. Nouet, H. Kirmse, W. Neumann, P. Komninou, and T. Karakostas, J. Appl. Phys. 110, 053715 (2011).

[26] S. Iwama, K. Hayakawa, and T. Arizumi, J. Cryst. Growth 56, 265 (1982).
[27] M. R. Ranade, F. Tessier, A. Navrotsky, and R. Marchand, J. Mater. Res. 16, 2824 (2001).

[28] C. A. Neugebauer and J. L. Margrave, Z. Anorg. Allg. Chem. 290, 82 (1957).

[29] Q. Guo and A. Yoshida, Jpn. J. Appl. Phys. 33, 2453 (1994).

[30] I. Zięborak-Tomaszkiewicz, J. Therm. Anal. Calorim. 83, 611 (2006).

[31] V. Davydov, A. Klochikhin, V. Emtsev, D. Kurdyukov, S. Ivanov, V. Vekshin, F. Bechstedt, J. Furthmüller, J. Aderhold, J. Graul, A. Mudryi, H. Harima, A. Hashimoto, A. Yamamoto, and E. Haller, Phys. Status Solidi B 234, 787 (2002).

[32] S. Plimpton, J. Comput. Phys. 117, 1 (1995).

[33] J. P. Perdew, K. Burke, and M. Ernzerhof, Phys. Rev. Lett. 77, 3865 (1996).

[34] P. E. Blöchl, Phys. Rev. B 50, 17953 (1994).

[35] P. Haas, F. Tran, and P. Blaha, Phys. Rev. B 79, 085104 (2009).

[36] L. Schimka, J. Harl, and G. Kresse, J. Chem. Phys. 134, 024116 (2011).

[37] J. Heyd, G. E. Scuseria, and M. Ernzerhof, J. Chem. Phys. 118, 8207 (2003).

[38] J. Heyd, G. E. Scuseria, and M. Ernzerhof, J. Chem. Phys. 124, 219906 (2006).

[39] X. Gonze, F. Jollet, F. A. Araujo, D. Adams, B. Amadon, T. Applencourt, C. Audouze, J.-M. Beuken, J. Bieder, A. Bokhanchuk, E. Bousquet, F. Bruneval, D. Caliste, M. Côté, F. Dahm, F. D. Pieve, M. Delaveau, M. D. Gennaro, B. Dorado, C. Espejo, G. Geneste, L. Genovese, A. Gerossier, M. Giantomassi, Y. Gillet, D. Hamann, L. He, G. Jomard, J. L. Janssen, S. L. Roux, A. Levitt, A. Lherbier, F. Liu, I. Lukačević, A. Martin, C. Martins, M. Oliveira, S. Poncé, Y. Pouillon, T. Rangel, G.-M. Rignanese, A. Romero, B. Rousseau, O. Rubel, A. Shukri, M. Stankovski, M. Torrent, M. V. Setten, B. V. Troeye, M. Verstraete, D. Waroquiers, J. Wiktor, B. Xu, A. Zhou, and J. Zwanziger, Comput. Phys. Commun. 205, 106 (2016).

[40] D. Segev, A. Janotti, and C. G. Van de Walle, Phys. Rev. B 75, 035201 (2007).

[41] N. Troullier and J. L. Martins, Phys. Rev. B 43, 1993 (1991).

[42] J. P. Perdew and Y. Wang, Phys. Rev. B 45, 13244 (1992).

[43] L. E. McNeil, M. Grimsditch, and R. H. French, J. Am. Ceram. Soc. 76, 1132 (1993).

[44] J. Serrano, A. Bosak, M. Krisch, F. J. Manjón, A. H. Romero, N. Garro, X. Wang, A. Yoshikawa, and M. Kuball, Phys. Rev. Lett. 106, 205501 (2011).

[45] I. Yonenaga, Y. Ohkubo, M. Deura, K. Kutsukake, Y. Tokumoto, Y. Ohno, A. Yoshikawa, and X. Q. Wang, AIP Adv. 5, 077131 (2015).

[46] D. Rodney, L. Ventelon, E. Clouet, L. Pizzagalli, and F. Willaime, Acta Mater. 124, 633 (2017).

[47] N. Lehto and S. Öberg, Phys. Rev. Lett. 80, 5568 (1998).

[48] M. S. Daw, Comput. Mater. Sci. 38, 293 (2006).

[49] J. P. Hirth and J. Lothe, Theory of Dislocations, 2nd ed. (Wiley, New York, 1982).

[50] H. J. Monkhorst and J. D. Pack, Phys. Rev. B 13, 5188 (1976).

[51] S. Kraeusel and B. Hourahine, Phys. Status Solidi A 209, 71 (2012). 
[52] R. Gröger, L. Leconte, and A. Ostapovets, Comput. Mater. Sci. 99, 195 (2015).

[53] L. Pizzagalli, J. Godet, and S. Brochard, Phys. Rev. Lett. 103, 065505 (2009).

[54] J. E. Northrup and J. Neugebauer, Phys. Rev. B 53, R10477 (1996).
[55] M. Bertelli, P. Löptien, M. Wenderoth, A. Rizzi, R. G. Ulbrich, M. C. Righi, A. Ferretti, L. Martin-Samos, C. M. Bertoni, and A. Catellani, Phys. Rev. B 80, 115324 (2009).

[56] H. Morkoç, Handbook of Nitride Semiconductors and Devices (Wiley-VCH, Darmstadt, 2008). 